\title{
Hypoxia-induced reduction of sVEGFR-2 levels in human colonic microvascular endothelial cells in vitro: Comparative study with HUVEC
}

\author{
CAREN JAYASINGHE ${ }^{1,2 *}$, NEKTARIA SIMIANTONAKI ${ }^{1,2^{*}}$, \\ ROMI MICHEL-SCHMIDT ${ }^{1}$ and CHARLES JAMES KIRKPATRICK ${ }^{1}$ \\ ${ }^{1}$ Institute of Pathology, Johannes Gutenberg University, Langenbeckstrasse 1, 55131 Mainz, Germany
}

Received August 1, 2008; Accepted September 15, 2008

DOI: 10.3892/ijmm_00000100

\begin{abstract}
The functionality of large-vessel endothelial cells, such as human umbilical vein endothelial cells (HUVEC), may differ significantly from that in the microvasculature. We established a method for the isolation of human colonic microvascular endothelial cells (HCMEC). Since colonic diseases are often accompanied by hypoxia we examined its effects on HCMEC of five individuals in comparison with HUVEC, with respect to the secretion of the soluble form of the two important vascular endothelial growth factor (VEGF) receptors, VEGFR-1 and 2. After dissociation by dispase/ collagenase of mucosal and submucosal tissue obtained from normal adult colon, HCMEC were isolated using CD31-coated magnetic beads and cultivated as monolayers. Subsequent characterization studies demonstrated the endothelial phenotype, including VEGFR-1 and 2 mRNA and protein expression. sVEGFR expression analyses were performed using ELISA. Under hypoxic conditions significantly enhanced levels of sVEGFR-1 on HUVEC were observed $(\mathrm{p}<0.001)$, while in HCMEC there was a markedly variable reaction to hypoxia, with cases of enhanced, unchanged and reduced expression. sVEGFR-2 was significantly decreased in HCMEC under hypoxia $(\mathrm{p}<0.001)$. In contrast, the responses of sVEGFR-2 levels to hypoxia in HUVEC were variable, that is, either unchanged or up-regulated. The different secretion profiles of sVEGFR-1 and 2 between HUVEC and HCMEC under normoxia and hypoxia underline the importance of using a functionally adequate and relevant microvasculature for in vitro studies of colonic diseases. The
\end{abstract}

Correspondence to: Dr Caren Jayasinghe and Dr Nektaria Simiantonaki, ${ }^{2}$ Present address: Institute of Pathology Klinikum Leverkusen, Am Gesundheitspark 11, 51137 Leverkusen, Germany E-mail: jayasinghe@klinikum-lev.de

E-mail: simiantonaki@yahoo.de

*Contributed equally

Key words: macrovasculature, microvasculature, hypoxia, sVEGFR-1, sVEGFR-2 homogeneously reduced sVEGFR-2 levels in hypoxic HCMEC provide evidence for a novel microvascular endothelium-specific biomarker in hypoxia-response processes.

\section{Introduction}

The endothelium, the monocellular lining of the blood vessel lumen strategically placed between the circulating blood and tissue, functions as a sensitive receptor-effector organ in an autocrine and paracrine manner in response to (patho) physiological stimuli $(1,2)$. Although endothelial cells present many common morphological features, they represent a structurally and functionally heterogeneous population of cells in different organs (3). Additionally, the endothelium of the macrovasculature, comprising the large arteries and veins, and that of the microvasculature (within small vessels), exhibit significant heterogeneity. Thus, microvascular but not macrovascular endothelium is involved in angiogenetic processes or in blood-tissue exchange of oxygen and nutrients. The diversity and complexity of endothelial cells necessitate the use of adequate vascular phenotypes for studying organspecific endothelium-tissue interactions in physiological and pathophysiological processes. Most of the data concerning endothelial cell function derive from the study of human umbilical vein endothelial cells (HUVEC), which are not just readily available, but also unproblematic with respect to their isolation and cultivation. However, these cells might not be an ideal model for studying specific endothelial functions, since they are cultured from aging, macrovascular vessels of embryonic origin.

The specificity of any tissue-associated microvasculature is determined by the environment, in which the endothelium is embedded and the functions it has to perform. The intestinal tract possesses a richly vascularized mucosa with a highly specialized microvasculature in relation to permanently varying $\mathrm{pO}_{2}$ values (4). This is attributed mainly to the special situation in the colon, where the mucosa is juxtaposed with the anaerobic lumen and as such is exposed to a uniquely physiological oxygen gradient. Additionally, the intestine is subject to large daily fluctuations of perfusion from the relatively low levels of blood volume during fasting to drastically increased perfusion after ingestion. Finally, 
barrier and absorptive functions of the gut between the luminal-vascular compartments can be regulated by oxygen. As a direct consequence, under physiological conditions the intestinal microvascular endothelium must be charactetized by resilience and robustness to altered levels of oxygenation.

In pathological intestinal disorders including infections, ischaemia, sepsis and inflammatory bowel disease, low oxygen tension or hypoxia often occurs (5-7). The initiating inflammation of all these pathological situations can be best described as a vascular response, where endothelial cells become activated, display increased permeability, enhanced immunoinflammatory cell adhesion and migration as well as angiogenesis (8). A major inducer of these processes is the vascular endothelial growth factor (VEGF), which mediates its biological effects by two related receptor tyrosine kinases, VEGFR-1 and VEGFR-2. VEGFR-2 is the major mediator of endothelial mitogenesis and survival as well as angiogenesis and microvascular permeability (9). VEGFR-1 functions as a negative regulator of VEGFR-2 signalling by sequestering VEGF. VEGFR1 is also required for the recruitment of haematopoietic precursors and migration of monocytes and macrophages. For both VEGF receptors a naturally occurring soluble form is known, sVEGFR-1 and $2(10,11)$. sVEGFR1 is a negative counterpart of the VEGF signalling pathway by sequestering VEGF and by binding and inactivating membrane-bound VEGFR-1 and 2. In contrast to sVEGFR-1, little is known about sVEGFR-2. Whether sVEGFR-2 can play a significant role in VEGF signalling, as has been described for sVEGFR-1, remains to be established.

The above considerations raise the question of whether the biological characteristics of HUVEC, including the response to hypoxia, differ significantly from highly specialized microvascular endothelial cells derived from the gut. In order to study the pathomechanisms of gut injury, we established a relatively simple and reproducible method for isolation of human colonic microvascular endothelial cells (HCMEC). Since intestinal diseases are often accompanied by hypoxic conditions we examined the effects of reduced oxygen tension on HCMEC in comparison with HUVEC, using the production of the soluble form of both receptors of VEGF, VEGFR-1 and 2, as parameters. We identified sVEGFR-2 as a novel sensitive factor, specific for microvascular endothelium in hypoxia-response processes.

\section{Materials and methods}

Isolation and culture of HCMEC. Human colonic microvascular endothelial cells were obtained from macroscopically normal portions of colon specimens surgically resected from patients who underwent colectomies for colon cancer. The present study was approved by the ethics committee of the University of Mainz and informed consent, as defined by the Helsinki Declaration, was obtained from each patient. Mucosa and submucosa from macroscopically normal colonic tissue were separated from the underlying musculature and cut into small fragments with scissors. After the removal of blood cells using PBS-BSA $0.1 \%$, the tissue was treated with dispase $\left(0.4 \%\right.$ at $4{ }^{\circ} \mathrm{C}$ for $18 \mathrm{~h}$; Sigma, Taufkirchen, Germany). After filtration the tissue was digested with collagenase type II $\left(0.2 \%\right.$, at $37^{\circ} \mathrm{C}$ for $40 \mathrm{~min}$; Serva,
Heidelberg, Germany) in a volume of $10 \mathrm{ml}$, followed by a further filtration step. The cell clumps were then repeatedly resuspended in PBS-BSA and filtered through a $100 \mu \mathrm{m}$ cell strainer. The cell suspension was then centrifuged for $5 \mathrm{~min}$ at $1500 \mathrm{Upm}$ and the cell pellet was resuspended in culture medium composed of MCDB131 with $15 \%$ fetal calf serum (FCS; Gibco, BRL, UK), Glutamax 1\% (Gibco), Fungizone $1 \%$ (Gibco) and Ciprobay 1\% (Bayer, Leverkusen, Germany) and seeded in gelatin-coated $(0.2 \%)$ tissue culture flasks. This mixed cell culture was cultivated at $37^{\circ} \mathrm{C}$ in a gas mixture of $5 \% \mathrm{CO}_{2}$ in air for $4 \mathrm{~h}$, followed by washing with PBS to remove all non-adherent cells. Fresh medium (Endothelial Growth Medium + Supplement Mix; Promocell, Heidelberg, Germany) was added and cells were cultivated as described above until subconfluence. The monolayer was subcultured 1:3 by trypsinization. Positive selection of HCMECs was achieved using magnetic beads ( $1 \mu \mathrm{m}$ diameter) coated with a mouse monoclonal antibody against CD31 (Dynal, Oslo, Norway) in passage one and two. In the third passage culture medium was changed to MCDB131 with $15 \%$ FCS, $1 \%$ glutamax, $1 \%$ penicillin/streptomycin, $2 \mathrm{ng} / \mathrm{ml} \mathrm{bFGF}$ (Sigma), $50 \mu \mathrm{g} / \mathrm{ml}$ Heparin (Sigma), and $50 \mu \mathrm{g}$ ECGS (Sigma).

Isolation and culture of HUVEC. HUVEC were isolated from human umbilical cords and cultured as previously described (12). For all experiments HUVEC in passage 2 were used.

Characterization of HCMEC. For immunfluorescence staining HCMECs were seeded on fibronectin-coated glass chamber slides (LabTec, Nunc, Wiesbaden, Germany) and grown to subconfluence. Cells were fixed with buffered $3.7 \%$ paraformaldehyde (15 min, room temperature) and permeabilized with $0.1 \%$ Triton X-100 (5 min, room temperature). Monoclonal mouse anti-human E-Selectin (Serva), monoclonal mouse anti-human CD31 (Dako, Hamburg, Germany), polyclonal rabbit anti-human Faktor VIII (Dako) monoclonal mouse anti-human sm-actin (Progen, Heidelberg, Germany) and monoclonal mouse anti-human cytokeratin (Dako) were used as primary antibodies. Alexa Fluor 488 goat anti-mouse and Alexa 546 goat anti-rabbit (Eugene, OR, USA) were used as secondary antibodies. Nuclear staining was performed with Hoechst 33342 (Sigma). Fluorescence labeled cells were covered with GelMount (Biomeda Corp/Natutec, Frankfurt, Germany).

For the cytokine and LPS stimulation experiments of E-selectin in HCMEC, endothelial cells were stimulated with $50 \mathrm{ng} / \mathrm{ml}$ LPS (Sigma), $20 \mathrm{ng} / \mathrm{ml} \mathrm{TNF-} \alpha$ (Sigma) and $0.5 \mathrm{ng} / \mathrm{ml}$ IL-1ß (Strathmann Biotech, Hamburg, Germany) for $6 \mathrm{~h}$.

Immunofluorescence. HCMECs and HUVECs were seeded onto fibronectin-coated glass chamber-slides (LabTek). After growing to subconfluence cells were exposed to the specific cell culture conditions, namely normoxia $\left(21 \% \mathrm{O}_{2}\right)$ and hypoxia $\left(3 \% \mathrm{O}_{2}\right)$. After exposure time, cells were fixed with buffered $3.7 \%$ paraformaldehyde (15 $\mathrm{min}$, room temperature) and permeabilized with $0.1 \%$ Triton X-100 (5 min, room temperature). Rabbit polyclonal anti-human FLT-1 (Santa Cruz, Heidelberg, Germany) and monoclonal anti-human KDR (Chemicon, Düsseldorf, Germany) were used as primary antibodies. Alexa Fluor 488 goat anti-mouse and 

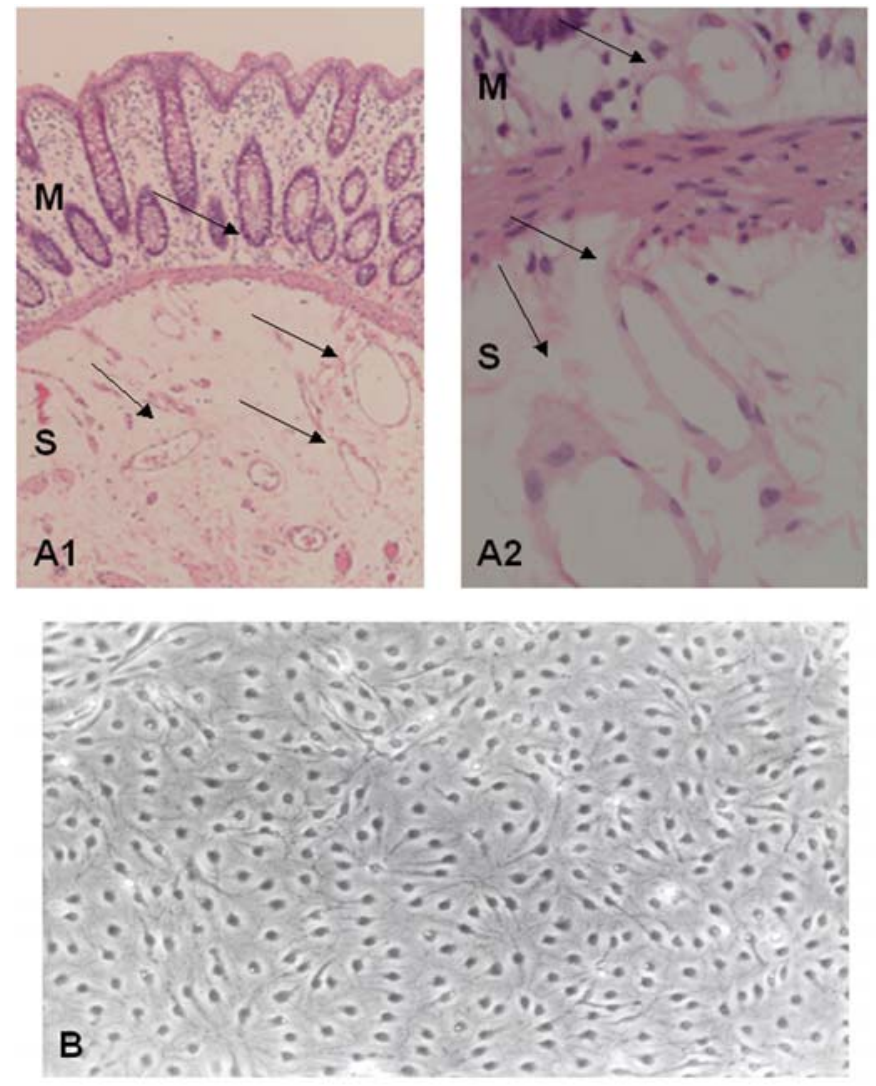

Figure 1. Morphology of colonic microvasculature in situ and in vitro. (A) Light microscopy of normal colonic mucosa (M) and submucosa (S) with abundant microvessels (arrow), from which HCMEC cultures are obtained (magnification: A1, 40x; A2, 200x). (B) Phase contrast microscopy of cultured HCMEC as confluent monolayer of closely associated fusiformshaped endothelial cells with rosette-like arrangement.

Alexa 546 goat anti-rabbit were used as secondary antibodies. Nuclear staining was performed with Hoechst 33342 (Sigma). Fluorescence labeled cells were covered with GelMount.

Semiquantitative Reverse Transcription-PCR. RNA isolation was performed using the RNeasy Kit (Qiagen, Hilden, Germany) in accordance with the manufacturer's protocols. B-actin and VEGFR-1 and 2 transcripts were analyzed using RT-PCR. RT was performed with the use of the Omniscript RT Kit (Qiagen) in accordance with the manufacturer's manual. As a template for the specific PCR reactions $2 \mu \mathrm{l}$ of the cDNAs-pool synthesized was used. To amplify the Bactin specific fragment, 574 base pair (bp), the primers 5'GAC CTG ACT GAC TAC CTC ATG A-3' (forward) and 5'-AGC ATT TGC GGT GGA CGA TGG AG-3' (reverse) were used. Amplification of the human VEGFR-1 specific fragment, $441 \mathrm{bp}$, was performed using the primers 5'-GCA CCT TGG TTG TGG CTG A-3' (forward) and 5'-GGT TTC GCA GGA GGT ATG GTG-3' (reverse). Amplification of the human VEGFR-2 specific fragment, $473 \mathrm{bp}$, was performed using the primers 5'-TAT GTC TAT GTT CAA GAT TAC-3' (forward) and 5'-AAG TTT CTT ATG CTG ATG CTT-3' (reverse). Amplification reactions were performed with the use of the Gene Amplification PCR System 2400 (Perkin Elmer, Norwalk, CT, USA) thermocycler. The
PCR products were separated on agarose gels (2\%), supplemented with ethidium bromide and analyzed by viewing under UV.

ELISA analysis. Levels of sVEGFR-1 and sVEGFR-2 were measured in cell lysates of HCMEC and HUVEC of 5 individuals cultivated under normoxic $\left(21 \% \mathrm{O}_{2}\right)$ and hypoxic $\left(3 \% \mathrm{O}_{2}\right)$ conditions over $24 \mathrm{~h}$. For the quantitative determination of human sVEGFR1 and 2 concentrations in cell lysates, commercially available sandwich ELISA assays (R\&D systems, Wiesbaden, Germany) were used according to the manufacturer's instructions. Cell lysates were prepared using a lysis buffer provided with the kit. The optic density of the color reaction was determined using a microplate ELISA reader set to $450 \mathrm{~nm}$.

Statistical analysis. Statistical analyses were performed using the Wilcoxon signed rank test. Significance was set at $\mathrm{p}<0.05$.

\section{Results}

Isolation and characterization of HCMEC. The colon is a highly vascularized organ, with a rich microvascular bed in the mucosa and submucosa. Fig. 1, A1 and A2 demonstrate the microvessels from normal human colon used to generate the HCMEC isolates. The isolation of HCMEC was carried out after various digestion and centrifugation steps and incubation with CD31-coated magnetic beads. The isolated endothelial cells were cultured in gelatine-coated flasks and grown to confluence within 6-8 days. The monolayer primarily consisted of closely associated cells with a fusiform shape as well as a rosette-like arrangement (Fig. 1B). The cultured cells were assayed for an endothelial-specific phenotype in the third passage of cultivation. In this passage enough cells were available for further experiments. To define the endothelial origin of HCMEC, expression analyses were performed using immunofluorescence staining. Detection of von Willebrand factor, which is stored in rod-shaped endothelial cell-specific storage organelles, the WeibelPalade bodies (13), showed the characteristic cytoplasmic, granular staining pattern (Fig. 2A). Detection of CD31, an integral membrane protein mediating cell-to-cell adhesions (14), demonstrated specific surface expression (Fig. 2A). In contrast, HCMEC did not recognize antibodies to whole-CK and sm-actin, a result which excludes the possibility of contamination with epithelial cells and subendothelial smooth muscle cells (data not shown). Culture impurity with fibroblasts can also be excluded because of the rapid overgrowth of this cell type in any mixed culture. In a following step of characterization, we investigated the regulated E-selectin expression on HCMEC. E-selectin is a member of the selectin family of endothelial cell adhesion molecules (15). Expression of E-selectin is tissue- and stimulusspecific, as it is generally expressed only in endothelial cells in response to induction by pro-inflammatory factors, such as tumor necrosis factor- $\alpha$ (TNF- $\alpha$ ), interleukin 1- $\beta$ (IL-1ß) and bacterial lipopolysaccharide (LPS), which makes E-selectin another key marker distinguishing endothelial cells from other cell types $(16,17)$. E-selectin is absent on quiescent 
A
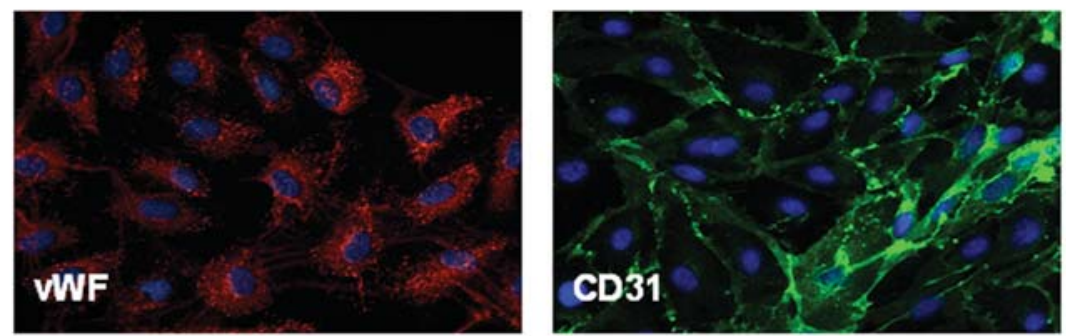

B
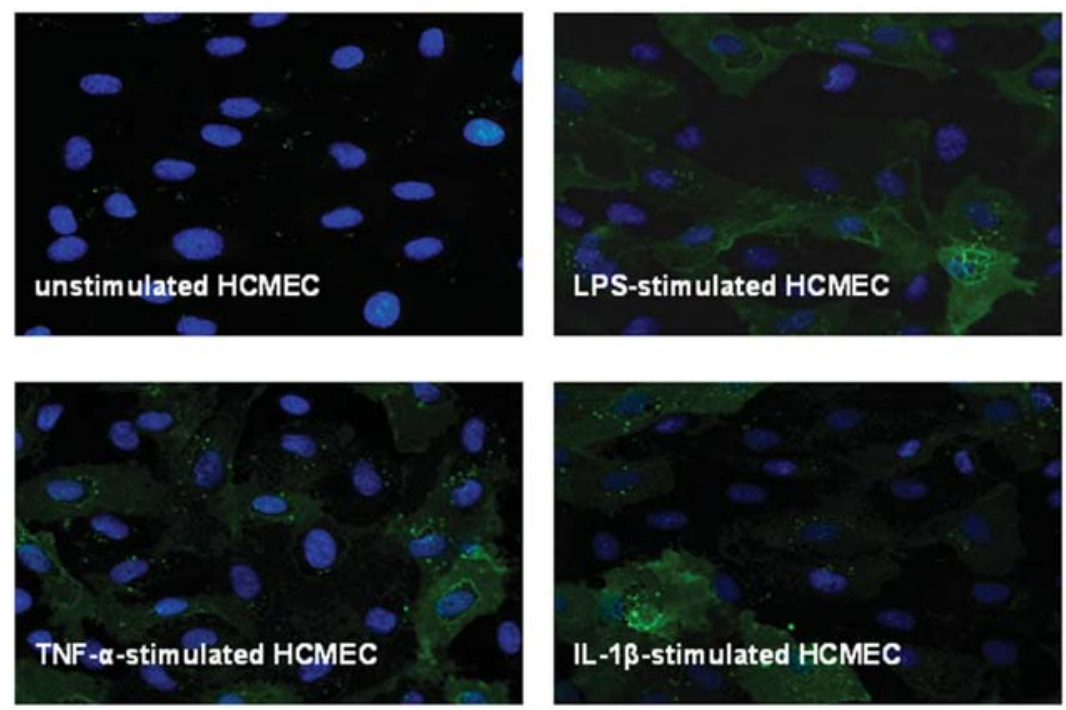

Figure 2. Characterization of HCMEC using immunofluorescence microscopy. (A) Detection of von Willebrand factor (vWF) showed specific cytoplasmatic, granular staining pattern and CD31 showed specific surface expression. (B) Cytokine and LPS inducible expression of E-selectin in HCMEC, whereas nonstimulated HCMEC do not express E-selectin, an inducible expression was found after stimulation of HCMEC with LPS, TNF- $\alpha$ and IL-1ß.

endothelium. As is shown in Fig. 2B, in comparison to nonstimulated HCMEC, which do not express E-selectin, an inducible expression was found after stimulation of HCMEC with TNF- $\alpha$, IL-1ß and LPS.

VEGFR-1 and 2 are expressed in HCMEC under normoxic and hypoxic conditions. To determine whether VEGFR-1 and VEGFR-2 were expressed on HCMEC under normoxic (21\% $\left.\mathrm{O}_{2}\right)$ and hypoxic $\left(3 \% \mathrm{O}_{2}\right)$ conditions, their transcription was examined using RT-PCR. As shown in Fig. 3A for both oxygen concentrations VEGFR-1 and 2 mRNA were expressed in HCMEC. No marked change in the VEGFR-1 and 2 mRNA levels were be found after $24 \mathrm{~h}$ of hypoxia. Overall, the expression levels of VEGFR-1 were lower in comparison with VEGFR-2, as seen by the weaker band. These data were congruent with the results obtained by immunofluorescence at the protein level (Fig. 3B).

sVEGFR-1 and 2 are differentially expressed in HUVEC and $H C M E C$. We examined the concentrations of sVEGFR-1 and sVEGFR-2 in cell lysates of HUVEC and HCMEC of five different donors and compared their amounts under normoxia and hypoxia using specific ELISA. Under hypoxic conditions a significant increase of sVEGFR-1 on HUVEC in all cases was observed (range: $350-400 \mathrm{pg} / \mathrm{ml}$ protein under normoxia versus 500-800 pg/ml under hypoxia; $\mathrm{p}<0.001$ )(Fig. 4A). The mean value of sVEGFR-1 levels was $366 \mathrm{pg} / \mathrm{mg}$ protein under normoxia and $640 \mathrm{pg} / \mathrm{mg}$ under hypoxia. In contrast, in HCMEC the sVEGFR-1 expression pattern was inconsistent, with a strongly pronounced range of variation of measured amounts (Fig. 4B). Whereas in one case the levels remained unchanged under normoxia and hypoxia $(380 \mathrm{pg} / \mathrm{ml}$ protein under normoxia versus $400 \mathrm{pg} / \mathrm{ml}$ under hypoxia), in two individuals hypoxia induced sVEGFR-1 reduction (1100 and $600 \mathrm{pg} / \mathrm{ml}$ protein under normoxia versus 750 and $400 \mathrm{pg} / \mathrm{ml}$ protein under hypoxia, respectively) and in two cases an increase (980 and $400 \mathrm{pg} / \mathrm{ml}$ protein under normoxia versus 1350 and $570 \mathrm{pg} / \mathrm{ml}$ protein under hypoxia, respectively) of sVEGFR-1 protein. Identical mean values of sVEGFR-1 levels for both oxygen concentrations were found $(692 \mathrm{pg} / \mathrm{ml}$ protein under normoxia versus $694 \mathrm{pg} / \mathrm{ml}$ under hypoxia).

In four of five investigated cases sVEGFR-2 was significantly reduced in HCMEC under hypoxia $(\mathrm{p}<0.001)$ (Fig. 5B). The mean values of sVEGFR2 levels were $2520 \mathrm{pg} / \mathrm{mg}$ protein under normoxia and $1782 \mathrm{pg} / \mathrm{mg}$ under hypoxia. Two hypoxic HUVEC cultures overexpressed sVEGFR-2 (700 
A

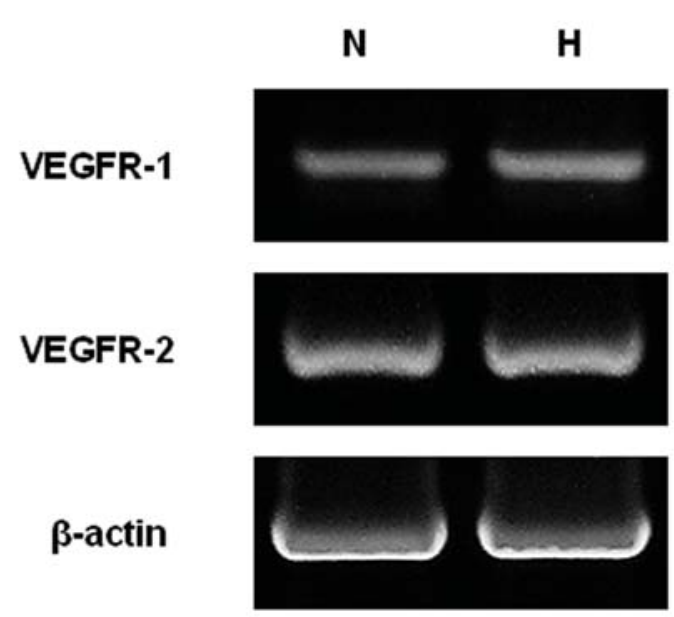

B

N

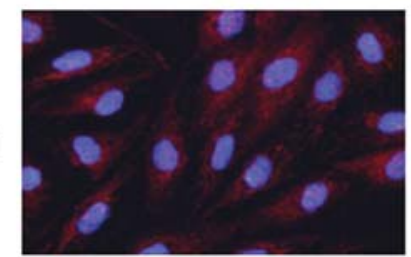

VEGFR-2

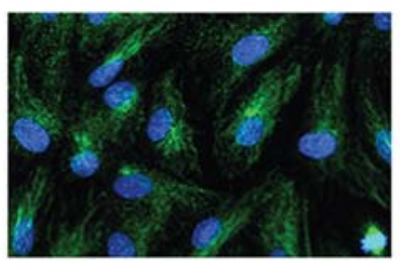

H
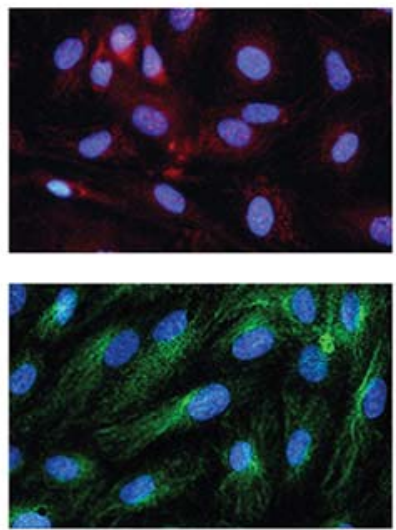

Figure 3. RT-PCR and immunofluorescence for VEGFR-1 and 2 expression in HCMEC under mormoxia (N) and hypoxia (H). (A) VEGFR-1 and 2 mRNA is detected in HCMEC under the two oxygen conditions, normoxia and hypoxia $\left(24 \mathrm{~h}, 3 \% \mathrm{O}_{2}\right)$ without significant differences in mRNA levels. The quality of the reaction was judged upon amplification of a specific B-actin fragment. (B) VEGFR-1 and 2 protein is detected in HCMEC without differences between normoxia and hypoxia.

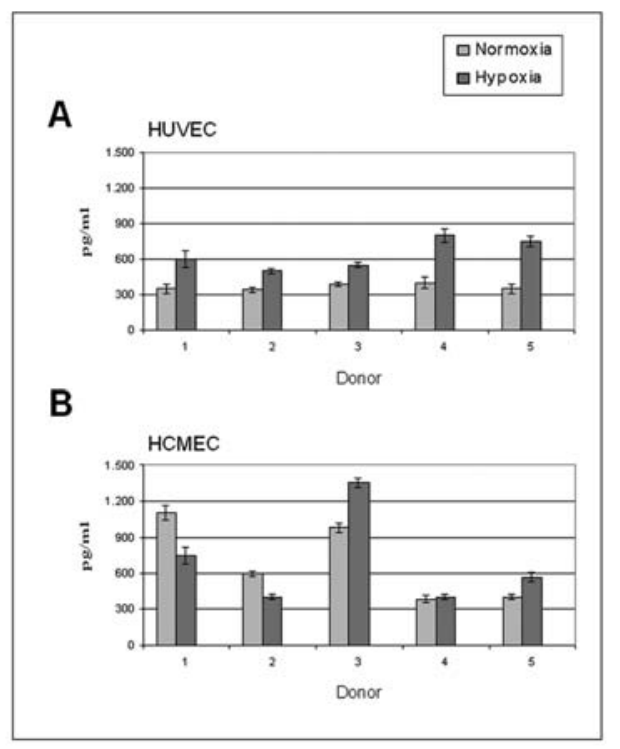

Figure 4. Determination of sVEGFR-1 concentrations by ELISA in HUVEC and HCMEC under normoxic conditions and after exposure to hypoxia $(n=3$, mean values \pm standard error). (A) After exposure of HUVEC to hypoxia sVEGFR-1 levels significantly increase ( $<<0.001)$. (B) In HCMEC the sVEGFR-1 level profiles are inconsistent. and $1800 \mathrm{pg} / \mathrm{ml}$ protein under normoxia versus 1200 and $2400 \mathrm{pg} / \mathrm{ml}$ protein under hypoxia, respectively) whereas three expressed the same levels under normoxic and hypoxic conditions (Fig. 5A). In the two endothelial types a strongly pronounced range of variation of measured amounts was found under both oxygen conditions (range: $700-1800 \mathrm{pg} / \mathrm{ml}$ protein for HUVEC and 1800-3400 pg/ml protein for HCMEC under normoxia; $1200-2400 \mathrm{pg} / \mathrm{ml}$ protein for HUVEC and 1000-2460 pg/ml protein for HCMEC under hypoxia). It is noteworthy that under normoxic conditions HCMEC exhibited significantly higher levels of sVEGFR-1 and 2 in comparison to HUVEC.

\section{Discussion}

In recent years heterogeneity between large and small vessel endothelium and between microvascular endothelial cells derived from different organs has become increasingly apparent. In vitro isolation and the study of endothelial cells under relevant conditions is an essential tool by which their function in physiology and pathophysiology can be explored. With this aim in view, we sought to isolate human microvascular endothelial cells (HCMEC) for studies of 


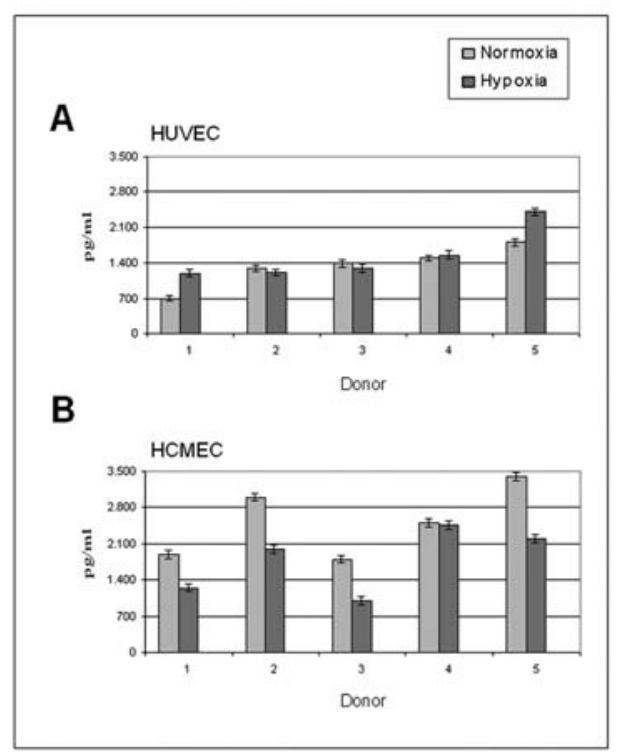

Figure 5. Determination of sVEGFR-2 concentrations by ELISA in HUVEC and HCMEC under normoxic conditions and after exposure to hypoxia ( $n=3$, mean values \pm standard error). (A) In hypoxic HUVEC the sVEGFR-2 levels are unchanged or increased. (B) After exposure of HCMEC to hypoxia sVEGFR-2 levels significantly decrease $(\mathrm{p}<0.001)$.

inflammatory and neoplastic diseases of the colon. By using CD31-coated magnetic beads microvascular endothelial cells were selectively isolated from a mixed primary culture of cells which are present in the gut mucosa and submucosa. The method is reproducible, relatively easy to apply and yielded endothelial cells with a high viability. Morphologically the endothelial cell colonies in culture, exhibited a fusiform appearance, which is also shown in microvascular endothelial cultures derived for other organs such as brain and esophagus $(18,19)$. The endothelial nature of the cells was demonstrated by positive immunostaining for phenotypic markers using antibodies to vWF and CD31, VEGFR-1 and 2 mRNA, protein expression, and by up-regulated expression of E-selectin after stimulation with LPS, TNF- $\alpha$ and IL-1ß.

In the following part of our study, the expression of the two VEGF receptors on HCMEC was examined especially under hypoxic conditions. Tissue hypoxia is a common feature of many inflammatory and ischemic diseases in the gut and plays a role in their pathogenesis. VEGFR-1 and 2 mRNA and protein were expressed in HCMEC under normoxic and hypoxic conditions. In the basal state, positive expression of VEGFR 1 and 2 mRNA in HCMEC has already been documented (20). In the present study in hypoxiaexposed HCMEC stable expression levels of these receptors were seen at the transcriptional level. This observation indicated a posttranscriptional regulation of both receptors independent of their promoter, although this observation requires quantitative confirmation. Previous reports demonstrating hypoxic mRNA induction of both VEGFRs have been contradictory. Waltenberger et al have shown an up-regulation of VEGFR-2 in hypoxia-exposed HUVEC and porcine aortic endothelial cells but without induction at the transcriptional level (21). In another study human dermal microvascular endothelial cells and macrovascular vein and artery endothelial cells, except HUVEC, responded to hypoxia with increased VEGFR-1 mRNA levels whereas VEGFR-2 transcript levels were elevated only in the microvascular endothelia (22). Gerber et al and Detmar et al have observed that hypoxia led to increased levels of VEGFR-1 mRNA in HUVEC and dermal microvascular endothelial cells but in contrast, VEGFR-2 mRNA levels were unchanged or slightly repressed $(23,24)$. During in vivo experiments with mice VEGFR-1 but not VEGFR-2 was induced by hypoxia in endothelial cells of the lung, heart, brain, kidney and liver (25). Notably, the VEGFR-1 gene is directly up-regulated by hypoxia via a hypoxia-inducible enhancer element located at its promoter region (23). In contrast, no hypoxia-inducible factor-1-related elements were found in VEGFR-2 promoter sequences, suggesting that the hypoxia-induced up-regulation of VEGFR-2 proceeds via an indirect mechanism. In this context, VEGF binding to the VEGFR-2 receptor tyrosine kinase results in an increase in VEGFR-2 gene transcription and protein expression (26). Additionally, the existence of a homeostatic system is known coordinating an appropriate VEGFR expression and controlled VEGF/VEGFR interaction (27). Thus, downregulation of receptor expression is coupled with upregulation of receptor mRNA expression followed by rapid replenishment of the cell surface with VEGF receptors and full recovery of responsiveness to VEGF. These data offer a very sensitive regulation system, which becomes more complicated by additive paracrine stimulation of the endothelium in vivo.

sVEGFR-1, a splice variant of the VEGFR-1 that lacks the transmembrane and cytoplasmic domains retains full VEGF binding potency and acts as a potent antiangiogenic molecule by binding circulating VEGF thus reducing the ligand binding to transmembrane and signalling receptors (10). In our study a consistent, significant increase of sVEGFR-1 in HUVEC under hypoxic conditions was observed. HUVEC originate from endothelial cells of the singular umbilical vein carrying oxygenated blood from the placenta to the fetus. Exposure of HUVEC to hypoxia induced VEGF mRNA and protein in a time-dependent manner with maximal levels observed at $48 \mathrm{~h}$ (28). Hypoxiainduced increase of sVEGFR-1 in HUVEC may be interpreted as a soluble antagonist form for VEGF leading to a decrease of free VEGF for contolled, continuously adequate vascular permeability to ensure sufficient oxygen and nutrient supply for the duration of the hypoxic stress. In contrast to HUVEC, in HCMEC a heterogeneous, donor-dependent sVEGFR-1 secretion pattern under hypoxia was observed. Colonic microvasculature is exposed to constantly varying $\mathrm{pO}_{2}$ values in response to large daily fluctuations of perfusion and as such is resilient and robust to altered levels of oxygenation (4). In this context, HCMEC show a marked donor-dependent sensitivity in regard to hypoxia-induced sVEFGR-1 secretion. Additional evidence for this statement is the strongly variable amount of sVEGFR-1 measured in the five investigated donors under the two test conditions, normoxia and hypoxia.

Ebos et al first reported the existence of a truncated protein of VEGFR-2 in mouse and human plasma (11). In contast to sVEGFR-1, little is known about sVEGFR-2. It appears likely that sVEGFR-2 is a product of ectodomain cleavage from the cell surface. The current generally accepted opinion 
is that VEGF binding and activation of VEGFR-2 leads to internalization and down-regulation of the receptor from the cell surface, which, in turn, leads to reduced sVEGFR-2 levels (29). An increase in circulating VEGF leads to a correlative decrease in sVEGFR-2 levels. In our study two hypoxic HUVEC cultures overexpressed sVEGFR-2 whereas three expressed the same levels under normoxic and hypoxic conditions. In contrast, in HCMEC cultures a consistent sVEGFR-2 reduction under hypoxia was seen. Wang et al have demonstrated that HCMEC internalize and degrade comparatively little VEGF and release a significantly greater fraction of VEGF into the medium in comparison to HUVEC (20). In this context, the hypoxia-induced, decreased sVEGFR-2 levels may reflect the significantly elevated release of VEGF from HCMEC compared to HUVEC under low oxygenation conditions. This observation reveals sVEGFR-2 as an important and sensitive hypoxia-related biomarker, specific for microvascular endothelial cells. Since microvascular but not macrovascular endothelium is involved in angiogenesis and in blood-tissue exchange of oxygen and nutrients, the observation that hypoxic stress leads to decrease of sVEGFR-2 only in microvascular endothelium may represent a novel mechanism for the release of a factor, namely sVEGFR-2, to regulate hypoxia-response processes. Which cellular mechanisms cause this sVEGFR-2 reduction and its direct consequences still have to be elucidated.

\section{Acknowledgements}

This study was funded by the state of Rhineland-Palatine, Germany within the scope of the general funds of the Institute of Pathology at the Johannes Gutenberg University in Mainz for personnel and consummable costs.

\section{References}

1. Kirkpatrick CJ, Wagner M, Hermanns I, Klein CL, Köhler H, Otto M, Van Kooten TG and Bittinger F: Physiology and cell biology of the endothelium: a dynamic interface for cell communication. Int J Microcirc Clin Exp 17: 231-240, 1997.

2. Galley HF and Webster NR: Physiology of the endothelium. Br J Anaesth 93: 105-113, 2004.

3. Risau W: Differentiation of endothelium. FASEB J 9: 926-933, 1995.

4. Taylor CT and Colgan SP: Hypoxia and gastrointestinal disease. J Mol Med 85: 1295-1300, 2007.

5. Giatromanolaki A, Sivridis E, Maltezos E, Papazoglou D, Simopoulos C, Gatter KC, Harris AL and Koukourakis MI: Hypoxia inducible factor 1alpha and 2alpha overexpression in inflammatory bowel disease. J Clin Pathol 56: 209-213, 2003.

6. Okuda T, Azuma T, Ohtani M, Masaki R, Ito Y, Yamazaki Y, Ito $\mathrm{S}$ and Kuriyama $\mathrm{M}$ : Hypoxia-inducible factor 1 alpha and vascular endothelial growth factor overexpression in ischemic colitis. World J Gastroenterol 11: 1535-1539, 2005.

7. Zinkernagel AS, Johnson RS and Nizet V: Hypoxia inducible factor (HIF) function in innate immunity and infection. J Mol Med 85: 1339-1346, 2007.

8. Danese S, Dejana E and Fiocchi C: Immune regulation by microvascular endothelial cells: directing innate and adaptive immunity, coagulation, and inflammation. J Immunol 178: 6017-6022, 2007.

9. Ferrara N, Gerber HP and LeCouter J: The biology of VEGF and its receptors. Nat Med 9: 669-676, 2003.

10. Kendall RL and Thomas KA: Inhibition of vascular endothelial cell growth factor activity by an endogenously encoded soluble receptor. Proc Natl Acad Sci USA 90: 10705-10709, 1993.
11. Ebos JM, Bocci G, Man S, Thorpe PE, Hicklin DJ, Zhou D, Jia X and Kerbel RS: A naturally occurring soluble form of vascular endothelial growth factor receptor 2 detected in mouse and human plasma. Mol Cancer Res 2: 315-326, 2004.

12. Simiantonaki N, Jayasinghe C and Kirkpatrick CJ: Effect of pro-inflammatory stimuli on tumor cell-mediated induction of endothelial cell adhesion molecules in vitro. Exp Mol Pathol 73: 46-53, 2002.

13. Reinders JH, de Groot PG, Sixma JJ and van Mourik JA: Storage and secretion of von Willebrand factor by endothelial cells. Haemostasis 18: 246-261, 1988.

14. DeLisser HM, Newman PJ and Albelda SM: Platelet endothelial cell adhesion molecule (CD31). Curr Top Microbiol Immunol 184: 37-45, 1993.

15. Tedder TF, Steeber DA, Chen A and Engel P: The selectins: vascular adhesion molecules. FASEB J 9: 866-873, 1995.

16. Wong D and Dorovini-Zis K: Regulation by cytokines and lipopolysaccharide of E-selectin expression by human brain microvessel endothelial cells in primary culture. J Neuropathol Exp Neurol 55: 225-235, 1996.

17. Haraldsen G, Kvale D, Lien B, Farstad IN and Brandtzaeg P: Cytokine-regulated expression of E-selectin, intercellular adhesion molecule-1 (ICAM-1), and vascular cell adhesion molecule-1 (VCAM-1) in human microvascular endothelial cells. J Immunol 156: 2558-2565, 1996.

18. Unger RE, Oltrogge JB, von Briesen H, Engelhardt B, Woelki U, Schlote W, Lorenz R, Bratzke H and Kirkpatrick CJ: Isolation and molecular characterization of brain microvascular endothelial cells from human brain tumors. In Vitro Cell Dev Biol Anim 38: 273-281, 2002.

19. Rafiee P, Ogawa H, Heidemann J, Li MS, Aslam M, Lamirand TH, Fisher PJ, Graewin SJ, Dwinell MB, Johnson CP, Shaker R and Binion DG: Isolation and characterization of human esophageal microvascular endothelial cells: mechanisms of inflammatory activation. Am J Physiol Gastrointest Liver Physiol 285: 1277-1292, 2003.

20. Wang D, Lehman RE, Donner DB, Matli MR, Warren RS and Welton ML: Expression and endocytosis of VEGF and its receptors in human colonic vascular endothelial cells. Am J Physiol Gastrointest Liver Physiol 282: 1088-1096, 2002.

21. Waltenberger J, Mayr U, Pentz S and Hombach V: Functional upregulation of the vascular endothelial growth factor receptor KDR by hypoxia. Circulation 94: 1647-1654, 1996.

22. Nilsson I, Shibuya M and Wennström S: Differential activation of vascular genes by hypoxia in primary endothelial cells. Exp Cell Res 299: 476-485, 2004.

23. Gerber HP, Condorelli F, Park J and Ferrara N: Differential transcriptional regulation of the two vascular endothelial growth factor receptor genes. Flt-1, but not Flk-1/KDR, is up-regulated by hypoxia. J Biol Chem 272: 23659-23667, 1997.

24. Detmar M, Brown LF, Berse B, Jackman RW, Elicker BM, Dvorak HF, and Claffey KP: Hypoxia regulates the expression of vascular permeability factor/vascular endothelial growth factor (VPF/VEGF) and its receptors in human skin. J Invest Dermatol 108: 263-268, 1997.

25. Marti HH and Risau W: Systemic hypoxia changes the organspecific distribution of vascular endothelial growth factor and its receptors. Proc Natl Acad Sci USA 95: 15809-15814, 1998.

26. Shen BQ, Lee DY, Gerber HP, Keyt BA, Ferrara N and Zioncheck TF: Homologous up-regulation of KDR/Flk-1 receptor expression by vascular endothelial growth factor in vitro. J Biol Chem 273: 29979-29985, 1998.

27. Wang D, Donner DB and Warren RS: Homeostatic modulation of cell surface KDR and Flt1 expression and expression of the vascular endothelial cell growth factor (VEGF) receptor mRNAs by VEGF. J Biol Chem 275: 15905-15911, 2000.

28. Namiki A, Brogi E, Kearney M, Kim EA, Wu T, Couffinhal T, Varticovski L, and Isner JM: Hypoxia induces vascular endothelial growth factor in cultured human endothelial cells. J Biol Chem 270: 31189-31195, 1995.

29. Ebos JM, Lee CR, Bogdanovic E, Alami J, Van Slyke P, Francia G, Xu P, Mutsaers AJ, Dumont DJ and Kerbel RS: Vascular endothelial growth factor-mediated decrease in plasma soluble vascular endothelial growth factor receptor-2 levels as a surrogate biomarker for tumor growth. Cancer Res 68: 521-529, 2008 . 